Article

\title{
Metal-to-Insulator Transitions in Strongly Correlated Regime
}

\author{
Jian Huang ${ }^{1, *}$, Loren Pfeiffer ${ }^{2}$ and Ken West ${ }^{2}$ \\ 1 Department of Physics and Astronomy, Wayne State University, Detroit, MI 48201, USA \\ 2 Department of Electrical Engineering, Princeton University, Princeton, NJ 08544, USA; \\ loren@princeton.edu (L.P.); kwest@princeton.edu (K.W.) \\ * Correspondence: jianhuang@wayne.edu; Tel.: +1-313-577-0564
}

Received: 30 November 2018; Accepted: 21 December 2018; Published: 26 December 2018

\begin{abstract}
Transport results from measuring ultra-clean two-dimensional systems, containing tunable carrier densities from $7 \times 10^{8} \mathrm{~cm}^{-2}$ to $\sim 1 \times 10^{10} \mathrm{~cm}^{-2}$, reveal a strongly correlated liquid up to $r_{s} \approx 40$ where a Wigner crystallization is anticipated. A critical behavior is identified in the proximity of the metal-to-insulator transition. The nonlinear DC responses for $r_{s}>40$ captures hard pinning modes that likely undergo a first order transition into an intermediate phase in the course of melting.
\end{abstract}

Keywords: electron-electron interaction; metal-insulator transition; strongly correlated phenomena; Wigner crystal; liquid-solid phase transition; non-Fermi liquid

\section{Introduction}

Anderson insulators [1] describe a quantum localization effect of non-interacting electrons due to random disorders. Such an insulator is expected to prevail in two-dimensional (2D) systems even in the low disorder limit [2], as long as the sample size $L$ and the electron coherence length $l_{\phi}$ are beyond the localization length $\zeta$. As far as the experimental results are concerned, Anderson localization provides a partial description of the transport behaviors because a $2 \mathrm{D}$ system can be a metal or an insulator as demonstrated extensively via the metal-insulator transition (MIT) [3,4] when the carrier density $n$ is reduced below a critical value $n_{c}$. While the disorder manifests in metals via the weak-localization (WL) [5] (with perturbation corrections from interaction [6,7]), it usually, as most results support, drives an Anderson insulator for $n<n_{\mathcal{c}}$ where the low- $T$ conduction is realized through hopping $[7,8]$ among the localized states. Thus, this scenario of MIT is characterized by the prominent disorder effect, with perturbation influences from interaction. However, this picture is radically altered if disorder is sufficiently reduced. This paper is aimed to demonstrate an MIT arising from strong interaction effects.

Strong interaction in the low disorder limit causes correlated electron states to emerge. Examples range from the long sought-after Wigner crystal (WC) [9] to the non-Fermi liquids [10]. The effect of interaction is reflected in a ratio $r_{s}=E_{e e} / E_{F}$ as a function of the carrier density $n: r_{s}=m^{*} e^{2} / \epsilon \hbar^{2} \sqrt{\pi n}$. $E_{e e}=e^{2} / \epsilon a$ is the Coulomb energy, $E_{F}=n \pi \hbar^{2} / m^{*}$ is the Fermi energy, and $m^{*}$ is the effective mass. Continuous increase of $r_{s}$ should eventually cause a liquid-solid phase transition (LSPT). Since a WC is always pinned by disorder [11], this LSPT manifests a distinctive MIT accompanied by a symmetry breaking. The predicted critical point for the LSPT is $r_{s}^{W C} \approx 37$ [12]. Therefore, it implies a considerable range for a strongly correlated non-Fermi liquid (non-FL) [10] and even possible intermediate phases (i.e. liquid crystals [13]) whose breakdown precedes the onset of a WC. Such an LSPT composes unknown manybody effects that are fundamentally important to a broad spectrum of strongly correlated phenomena. However, rigorous demonstration of a WC state and the LSPT are still lacking. 
Realization of a large $r_{s}$ in a zero magnetic field requires the lowering of $n$ which necessarily reduces both $E_{e e}$ and $E_{F}$. The rising effect from disorder at dilute $n$ tends to drive an Anderson localization (or percolation transition depending on the nature and the amount of the disorders) and breaks long-range orders. Most systems do not have sufficiently low disorders so that they become Anderson insulators (or a glass or mixed phases) before reaching $r_{s}^{W C}$. This is evidenced by the activated low- $T$ conductivity $\sigma(T)=\sigma_{0} \exp \left(-T^{*} / T\right)^{1 / \gamma}(\gamma=2,3)$ occurs whenever $r_{s}$ is raised beyond 20. Consequently, the value of $n_{c}\left(\right.$ or $\left.r_{s}^{c}\right)$ is sensitive to the disorder levels [14,15].

Adoption of ultra-clean 2D systems is crucial to avoid disorder-driven localization at large $r_{s}$. For this study, the samples are $p$-type 2D systems in (100) GaAs/AlGaAs heterojunction-insulated-gate field-effect-transistors (HIGFETs). The carrier density $p$ is tunable between $7 \times 10^{8} \mathrm{~cm}^{-2}$ to $3 \times 10^{10} \mathrm{~cm}^{-2}$, corresponding to $70>r_{s}>25$. Without any intentional doping, the carriers accumulate at the hetero-interface in response to a voltage bias on the metal gate beyond a threshold $\left(V_{t h} \sim-1.3 \mathrm{~V}\right)$. The sample contains a $6 \mathrm{~mm} \times 0.8 \mathrm{~mm}$ Hallbar fabricated with a self-align technique $[16,17]$. Ohmic contacts are realized via annealing AuBe alloys. Measurement is made inside a dilution refrigerator. The carrier density $p$ is determined by the quantum Hall measurement. The carrier mobility $\mu$ reaches $1.04 \times 10^{6} \mathrm{~cm}^{2} / \mathrm{V} \cdot \mathrm{s}$ at $p=1.2 \times 10^{10} \mathrm{~cm}^{-2}$.

\section{Results}

An MIT associated with an interaction-driven phase transition should occur around $r_{s}^{W C}$. The actual $r_{s}^{W C}$ is not yet well determined though a somewhat higher value is anticipated considering the thermal, quantum, and disorder fluctuations. The presentation of the results starts with the metallic phase which indeed confirm a conductor up to $r_{s}^{c} \sim 40 \sim r_{s}^{W C}$ where signatures supporting a critical behavior emerge. The critical $r_{s}$ is found to settle around $r_{s}^{W C}$, showing little dependence on the samples. At $r_{s}>r_{s}^{c}$, the transport is nonactivated over a range of $T$ which contrasts the disorder-driven localization. Finally, the nonlinear response for $r_{s}>r_{s}^{c}$ reveals prominent pinning modes characterized by a large translational correlation length $\xi$. The dependence on the temperature and the external bias display a behavior supporting an LSPT mediated by an intermediate phase.

\subsection{Metal Behaviors at Large $r_{s}$ and a Critical Behavior}

Though most 2D metallic behaviors are well described by the Fermi liquid (FL) [18], the breakdown of the FL is inevitable in both disorder- and interaction-dominated regimes. Indications of non-FL states are mostly documented for the metallic states in the proximity of $n_{c}$ corresponds to a $r_{s} \sim 10-20$. Evidence includes $T$-dependence of the resistivity [19-22], the magnetoreistance $\left(\rho_{x x}\right)$ in a parallel $B_{\|}$field [22,23], and the Coulomb drags [24], etc. The suppression of disorder reduces $r_{s}^{c}$ and opens up a range for the non-FL regime. Verification of a metallic state persisting up to the anticipated $r_{s}^{W C}$ is crucial to establish a LSPT. The results below confirm a metal state up to a $r_{s}$ that is in fact approximately the $r_{s}^{W C}$.

The metallic state is determined by the $T$ dependence of the resistivity $\rho(T)$ measured with a four-probe AC lock-in technique. Figure 1a shows the $\rho(T)$ for $p=5.9 \times 10^{9} \mathrm{~cm}^{-2}$ (or $r_{s}=37$ ) which unambiguously confirms a metal behavior: $\rho(T)$ is below the quantum of conductance $h / e^{2}$ and $d \rho / d T \sim 3 \mathrm{k} \Omega / 100 \mathrm{mK}$ has a positive sign below a characteristic temperature $T_{\max }$ where $\rho(T)$ peaks. $T_{\max } \sim 125 \mathrm{mK}$ is $\ll T_{F}=E_{F} / k_{B} \sim 400 \mathrm{mK}$. Figure $1 \mathrm{c}$ confirms the same metal behavior in a different sample with a carrier density of $p=5.2 \times 10^{9} \mathrm{~cm}^{-2}$ (or $r_{s}=40$ ) down to $T=4 \mathrm{mK}$. Note that the ac current excitation is kept under $0.5 \mathrm{nA}$ so that Joule heating at $<1 \times 10^{-17} \mathrm{~W}$ is negligible. $\rho(T)$ is studied for multiple carrier densities and the characteristics related to a critical behavior are found through the $r_{s}$ dependence of $T_{\max }$. 

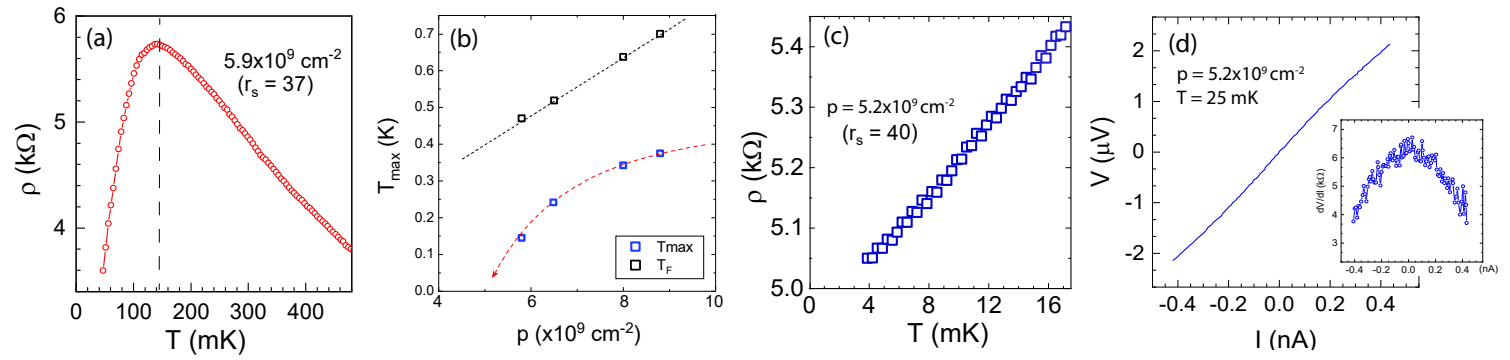

Figure 1. (a) $T$ dependence of the sheet resistance for $r_{s}=37$ down to $40 \mathrm{mK}$ and (b) The $p$ dependence of $T_{\text {max }}$ in comparison to $E_{F}$. (c) $\rho(T)$ for $r_{S}=40$ down to $4 \mathrm{mK}$. (d) DC-IV for $p=5.2 \times 10^{9} \mathrm{~cm}^{-2}$ measured at $25 \mathrm{mK}$ for a current range between 0 and $\pm 0.4 \mathrm{nA}$. Inset: $d V / d I$ based on the IV result.

$T_{m a x}$ is sometimes associated with a renormalized Fermi energy $E_{F}^{*}(p)$ [11]. As shown in Figure $1 c$ where the $p$ or $\left(r_{s}\right)$ dependence of $T_{\max }$ is shown, $T_{\max }$ is indeed below $T_{F}=E_{F} / k_{B}$ which is qualitatively correct from the stand point of a strong renormalization [10]. However, the nonlinear fall of $T_{\max }$ with decreasing $p$ is far too rapid to be accounted for by FL renormalization: a nearly three-fold drop from $p=8.5$ to $5.8 \times 10^{9} \mathrm{~cm}^{-2}$. In fact, the trend of diminishing $T_{\max }$ suggests a critical behavior. A nonlinear three-free-parameter fit to a scaling $T_{\max }\left(r_{s}\right)=T_{0}\left(r_{s}^{c}-r_{s}\right)^{\alpha}$ produces $T_{0}=116.4 \pm 5.3 \mathrm{mK}$, $r_{s}^{c}=41.08 \pm 0.6$, and $\alpha=0.488 \pm 0.25$, with a $R^{2} \approx 0.9987 . r_{s}^{c} \sim 41$ corresponds to a critical density $p_{c} \sim 5 \times 10^{9} \mathrm{~cm}^{-2}$. Carrier density dependent scaling has been reported in Si-MOSFETs where a similar exponent is found [25]. Note that the value of $m^{*}=0.35 m_{0}$ used for extracting $r_{s}$ values is based on the cyclotron resonant studies [26,27] obtained for similar carrier densities.

In addition, the DC-IV reveals a nonlinear response incompatible with a FL. As shown in Figure 1d, the DC-IV for $r_{s}=40$ (or $p=5.2 \times 10^{9} \mathrm{~cm}^{-2}$ ) is nonlinear within a current window of just $\pm 0.4 \mathrm{nA}$. The derivative $d V / d I$ shown in the inset figure varies continuously from $6.3 \mathrm{k} \Omega$ at $I=0$ to $3.5 \mathrm{k} \Omega$ at $I= \pm 0.4 \mathrm{nA}$. The nonlinearity is easily resolved down to tiny drives below $I \sim 0.1 \mathrm{nA}$ where the Joule heating dissipates negligibly at $<5 \times 10^{-17} \mathrm{~W}$. Nonlinear DC response associated with interaction has been extensively reported for both the insulating regime, in connection to the finite pinning effects [28], as well as in the metallic regime, as identified in mesoscopic systems in a B-field [29]. As shown later for larger $r_{s}$, the nonlinear DC-IV becomes increasingly prominent and eventually manifests as a threshold similar to the pinned charge density waves (CDWs) [30]. The weak nonlinear response indicates the onset of a possible intermediate phase which is further discussed later in this report.

\subsection{Critical Density and the Influence of the Disorder}

As confirmed by most experiments $[3,4,11,14,31,32]$, the $r_{s}^{c}$ in more disordered systems is well below $r_{s}^{W C}$ and it varies with the changes in the disorder levels. This is, however, contrasted by results obtained from ultra-clean systems where the $r_{s}^{c}$ observed among various samples tends to settle approximately at a large $r_{s}^{c} \sim 40$. Figure 2a shows $\rho(T)$, on semi-logrithimic scales, obtained from one of the samples for a number of carrier densities from 1.2 to $12.5 \times 10^{9} \mathrm{~cm}^{-2}$. Note that the four curves from $10 \mathrm{~K}$ to $300 \mathrm{mK}$ (in black) are obtained in a 3-Helium cryostat and $1 \mathrm{~K}$ to $30 \mathrm{mK}$ results are obtained in a dilution refrigerator. For $p \leq 4 \times 10^{9} \mathrm{~cm}^{-2}$ (blue curve), only insulators are found. $p_{c}$ is estimated as $\sim 4.5 \times 10^{9} \mathrm{~cm}^{-2}$ ( or $r_{s}^{c} \sim 43$ assuming $m^{*}=0.35 m_{0}$ ) which is in good agreement with the $T_{\max }$ extrapolation [Figure 1b]. Consistent $p_{c}$ has been found in multiple HIGFET samples from four different wafers. Some of the results are reported in Refs. $[19,20]$. The histogram of $p_{c}$ shown in the inset figure is based on results collected from seven different samples. 

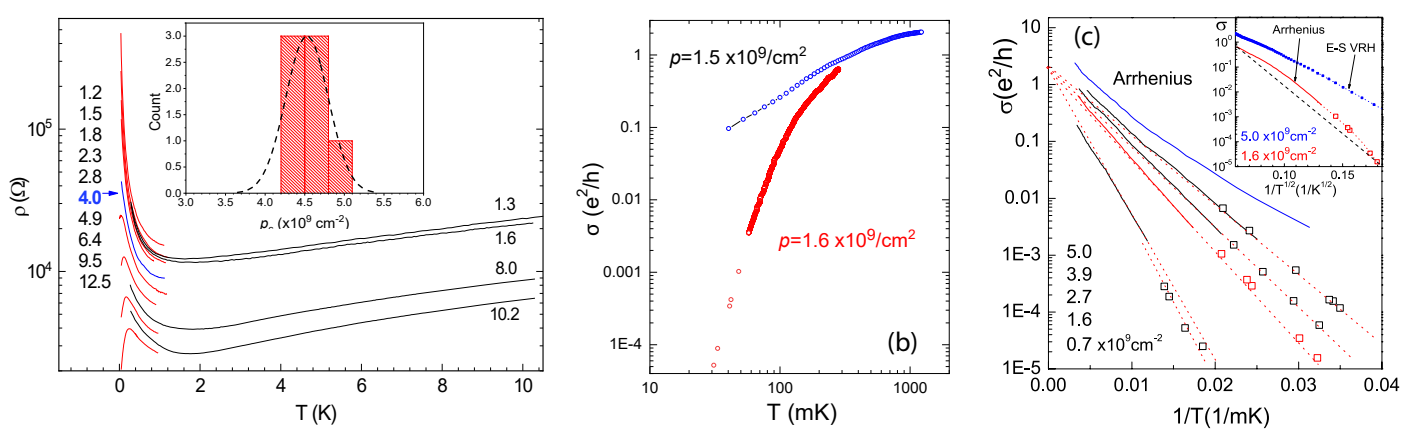

Figure 2. (a) $T$ dependence of the resistivity $\rho(T)$ for a carrier density range from $7 \times 10^{8} \mathrm{~cm}^{-2}$ to $1.25 \times 10^{10} \mathrm{~cm}^{-2}$. (b) Comparison of the nonactived conductance (in blue) of a clean system to the activated hopping conductance (in red) of a more disordered system for $p \sim 1.5 \times 10^{9} \mathrm{~cm}^{-2}$. (c) The nonactivated behavior is turned into an activated behavior as a result of increase of disorders. $p$ is from $7 \times 10^{10} \mathrm{~cm}^{-2}$ to $5 \times 10^{9} \mathrm{~cm}^{-2}$. Solid lines are the AC results and the scattered points are the DC results. Dotted lines are a guide to the eye. Inset: comparison between an Arrhenius hopping and the Efros-Shklovskii variable range hopping.

Meanwhile, the transport of the insulator draws a distinction from the activated behavior. Figure $2 \mathrm{~b}$ displays on double-logarithmic scales the qualitative differences in the conductivity $\sigma(T)$ $(=1 / \rho)$ between the non-activated $T$ dependence in an ultra-clean case, with $p=1.5 \times 10^{9} \mathrm{~cm}^{-2}$, and the activated $T$ dependence in a more disordered case, with $p=1.6 \times 10^{9} \mathrm{~cm}^{-2}$. Though similar values of $\sigma(T)$ are found for both cases beyond $200 \mathrm{mK}$, an activated behavior is only observed for the more disordered system whose $\sigma(T)$ plummets by more than three orders of magnitude compared to the clean case. A power-law fit, $\sigma(T) \propto T^{\alpha}$, to the low $T$ nonactivated results produces an $\alpha \sim 1.35$. These striking differences are also observed in other studies $[19,20]$.

The influences of the disorder is demonstrated in Figure 2c. When extra disorders are introduced, through LED illumination in our case, the nonactivated behavior diminishes and an Arrhenius activated transport is recovered as shown by the $\sigma(T)$ versus $1 / T$ on semi-logarithmic scales. Consequently, $p_{c}$ rises substantially to nearly $p=9.2 \times 10^{9} \mathrm{~cm}^{-2}$. A comparison to the variable range hopping $(\mathrm{VRH})$ is provided in the inset of Figure 2c.

\subsection{Nonlinear Responses and the Liquid-Solid Phase Transition}

Detection of collective modes of possible WCs has been carried out in both the quantum Hall regime, with respect to pinning [28,33-35], resonant absorption (via RF (Radio frequency), microwaves, acoustic waves [36-39], and tunneling [40]), as well as in a zero magnetic field (B) [14,41-43]. Nevertheless, a WC phase is not yet well supported for a number of reasons. First, the correlation length $\xi$ is rather small $(\leq 1 \mu \mathrm{m})$ and decays exponentially with increasing $T$. As the pinning strength scales with $\xi[44,45]$, it explains why the observed differential resistance is not as robust as expected. These findings favor an intermediate or a mixture of liquid and solid phases. Second, the reported melting $[37,38,40]$ is reflected via a smooth crossover $[28,37,38,40]$, lacking a singularity that reflects the changes in the free energies across the phase boundary [46]. Some of the experimental challenges can be understood by the sensitive nature of the WC on the disorder, $T$, the magnetic field (for WC studies in the fractional quantum Hall regime), and even the experimental conditions. Suppression of disorder is key to not only reducing the chances of glass [47-49] or mixed phases, but also to maintaining an accessible critical temperature $T_{\mathcal{c}}$ which can be suppressed substantially by disorder fluctuations [50-52]. The results presented below are based on the DC response as a probe to both pinning and melting effects.

Figure 3a shows the DC-IV obtained for $r_{s} \sim 50$ where a contrast is found between two close-by temperatures: 32 and $43 \mathrm{mK}$. The DC response at $43 \mathrm{mK}$ or higher $T$ (not shown) is approximately linear up to $50 \mathrm{pA}$ and is invariant when switching the leads to different orientations. This finding 
is consistent with the previous results $[28,34]$ interpreting an isotropic liquid. On the other hand, the DC-IV exhibits a nonlinear response at $32 \mathrm{mK}$, indicating the presence of pinning. The differential resistance $r_{d}$ measured with a current $I$ below $5 \mathrm{pA}$ is $\sim 18 \mathrm{M} \Omega . r_{d}$ approaches a linear behavior with increasing $I$ and reaches the same $r_{d}$ as for the $43 \mathrm{mK}$ case. Assuming collective pinning of $N=p \xi^{2}$ carriers by a periodic potential after the CDW model, previous studies $[28,34]$ adopted the following equation to estimate $\xi$ :

$$
I(E) \propto \sinh (N e E a / T) \exp \left[-\left(2 N e E_{t h} a / \pi T\right)\right]
$$

$E_{t h}=V_{t h} / L$ ( $L$ is the length between the voltage contacts) is the threshold electric field. At the threshold, $e E_{t h} a$ is $\sim 0.01 \mu \mathrm{eV}$ (or $0.1 \mathrm{mK}$ ) $\ll k_{B} T$, confirming a collective nature. As shown in Figure 3a, fitting the $32 \mathrm{mK}$ curve to equation (1) produces $N \sim 200$ or $\xi \sim 14 a \sim 1.4 \mu \mathrm{m}$ at $R^{2}=0.9995$. Nevertheless, this fitting makes an assumption that $N$ is only affected by $T$ and is independent of $V$. This, however, is incorrect if a melting instead of depinning occurs. Note that these results associated with soft pinning are qualitatively consistent with the previous results [28].
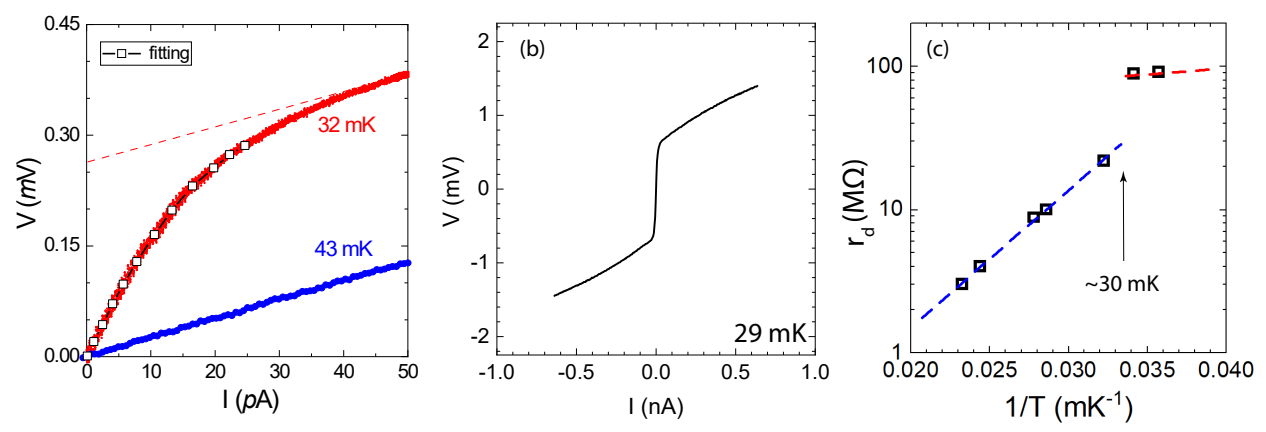

Figure 3. (a) Nonlinear and linear DC-IV for $r_{s}=53$ at 32 and $43 \mathrm{mK}$ within a current window up to $50 \mathrm{pA}$. Dashed line is a guide for the eye. (b) Threshold DC-IV obtained at $29 \mathrm{mK}$ for the same $r_{s}$ (c) Piecewise behavior of $r_{d}(T)$ displayed in $\log \left(r_{d}\right)$ vs. $1 / T$.

Figure $3 \mathrm{~b}$ shows the DC-IV obtained for the same $r_{s}$ at $29 \mathrm{mK}$ where a threshold behavior appears. The sub-threshold pinning increases substantially as indicated by $r_{d}=d V / d I \sim 100 \mathrm{M} \Omega$. As shown by previous studies $[28,34], \xi$ is estimated by balancing the total potential energy $N \epsilon$ and the pinning energy $-(1 / 2) \kappa a . \kappa=0.245 e^{2} p^{3 / 2} / 4 \pi \epsilon_{0} \epsilon$ is the sheer modulus and $N=p \xi^{2}$ is the particle numbers in the domain. For our case, this yields $N \geq 10^{5}$ or $\xi \geq 50 \mu \mathrm{m}$. As clearly shown by the results below by the general nonlinear IVs up to $43 \mathrm{mK}, \xi$ decreases with increasing $V$. Therefore, the estimated $\xi$ reflects a lower limit.

The pinning effect depends on both $T$ and the external bias. Thus, to probe a thermal melting, the external bias signal must be limited to the lowest levels. Figure $3 \mathrm{c}$ shows the $r_{d}$ measured with $I \leq 0.5 \mathrm{pA}$ at several temperatures. $r_{d}(T)$ exhibits a piecewise behavior, supporting a phase transition at $T_{c} \sim 30 \mathrm{mK}$ where a sharp jump of nearly $60 \mathrm{M} \Omega$ appears. The jump can be viewed as a potential energy loss if $\xi$ is suddenly reduced. Because a pinned WC is characterized by the potential energy, $r_{d}$ scales with $\xi$. By limiting $I$ below $0.5 \mathrm{pA}$, the nonlinear effect related to the level of excitation diminishes. Thus, this result implies a piecewise $T$ dependence of $\xi$ which is proportional to the energy within a WC domain. A recent Monte Carlo study indeed predicts a discontinuity in the free energy across the phase boundary between a WC and a quantum hexatic phase [51]. As a finite pinning [53] is retained above $T_{\mathcal{C}}$, this transition indeed ends up in an intermediate phase instead of a liquid. Melting through intermediate phases has been modeled for hexatics [51,54-57], stripes, bubbles, and microemulsions [58]. For the $T$ dependence of $r_{d}$ above $T_{\mathcal{c}}$ (or for $1 / T \leq 0.033$ in Figure $3 c$ ), $r_{d}$ falls exponentially with increasing $T$, which is qualitatively consistent with the exponential $T$ dependence of $\xi$ predicted for the hexatic phases $[52,55,56]$. 


\section{Discussion}

In addition to a thermal melting, a phase transition can also be triggered by the external bias. The threshold in the DC-IV behavior for $r_{s}>r_{s}^{W C}$ at $T<T_{c}$ is often associated with the depinning of a WC which is perhaps influenced by the sliding CDW picture. However, there is possibly a more energetically-favorable alternative - the onset of an intermediate phase whose correlation length decreases with increasing bias until reaching a liquid phase. The critical behavior demonstrated in the $r_{s}$ dependence of $T_{\max }$ suggests a relevant energy scale of which the origin needs to be better understood. $r_{s}^{c}$ being larger than the estimated $r_{s}^{W C}$ is likely due to the finite disorder effect which can not be quantified at this point. The discontinuity [46] in the thermal melting is consistent with a recent theoretical work [51]. $T_{c} \sim 30 \mathrm{mK}$ is only $25 \%$ of the classical melting point $E_{e e} / 137$. This may be an indication to the fact that a quantum melting differs from the classical process such as the Kosterlitz-Thouless transition [59] due to an extra degree of freedom. In this preliminary report, several important questions are left unanswered. Subjects such as the nature of the intermediate phase, the free energy differences between the phases, effects from the spin-orbit coupling, etc. need further exploration.

Author Contributions: Conceptualization, J.H.; methodology, J.H.; software, J.H.; validation, J.H.; formal analysis, J.H.; investigation, J.H.; resources, J.H.; data curation, J.H.; writing-original draft preparation, J.H.; writing-review and editing, J.H.; visualization, J.H.; supervision, J.H.; project administration, J.H.; funding acquisition, J.H.", Sample and crystal growth: L.P. and K.W.

Funding: The work at Wayne State University is supported by NSF under DMR-1410302, The work at Princeton University was funded by the Gordon and Betty Moore Foundation through the EPiQS initiative Grant GBMF4420, and by the National Science Foundation MRSEC Grant DMR 1420541.

Acknowledgments: The authors are grateful for the helpful discussions with Myriam Sarachik and David Ceperley.

Conflicts of Interest: The authors declare no conflict of interest.

\section{Abbreviations}

The following abbreviations are used in this manuscript:

MDPI Multidisciplinary Digital Publishing Institute

DOAJ Directory of open access journals

TLA Three letter acronym

LD linear dichroism

\section{References}

1. Anderson, P.W. Absence of Diffusion in Certain Random Lattices. Phys. Rev. 1958, 109, 1492-1505. [CrossRef]

2. Abrahams, E.; Anderson, P.W.; Licciardello, D.C.; Ramakrishnan, T.V. Scaling Theory of Localization: Absence of Quantum Diffusion in Two Dimensions. Phys. Rev. Lett. 1979, 42, 673-676. [CrossRef]

3. Kravchenko, S.V.; Kravchenko, G.V.; Furneaux, J.E.; Pudalov, V.M.; D’Iorio, M. Possible metal-insulator transition at $B=0$ in two dimensions. Phys. Rev. B 1994, 50, 8039-8042. [CrossRef]

4. Kravchenko, S.V.; Mason, W.E.; Bowker, G.E.; Furneaux, J.E.; Pudalov, V.M.; D'Iorio, M. Scaling of an anomalous metal-insulator transition in a two-dimensional system in silicon at $B=0$. Phys. Rev. B 1995, 51, 7038-7045. [CrossRef]

5. Bergmann, G. Weak localization in thin films: a time-of-flight experiment with conduction electrons. Phys. Rep. 1984, 107, 1-58. [CrossRef]

6. Altshuler, B.L.; Aronov, A.G.; Lee, P.A. Interaction Effects in Disordered Fermi Systems in Two Dimensions. Phys. Rev. Lett. 1980, 44, 1288-1291. [CrossRef]

7. Efros, A.L.; Shklovskii, B.I. Coulomb gap and low temperature conductivity of disordered systems. J. Phys. C Solid State Phys. 1975, 8, L49. [CrossRef]

8. Mott, N.F. Conduction in non-crystalline materials. Philos. Mag. 1969, 19, 835-852. [CrossRef]

9. Wigner, E. On the Interaction of Electrons in Metals. Phys. Rev. 1934, 46, 1002-1011. [CrossRef]

10. Varma, C.; Nussinov, Z.; Van Saarloos, W. Singular or non-Fermi liquids. Phys. Rep. 2002, 361, 267-417. [CrossRef] 
11. Abrahams, E.; Kravchenko, S.V.; Sarachik, M.P. Metallic behavior and related phenomena in two dimensions. Rev. Mod. Phys. 2001, 73, 251-266. [CrossRef]

12. Tanatar, B.; Ceperley, D.M. Ground state of the two-dimensional electron gas. Phys. Rev. B 1989, 39, 5005-5016. [CrossRef]

13. Wright, D.C.; Mermin, N.D. Crystalline liquids: The blue phases. Rev. Mod. Phys. 1989, 61, 385. [CrossRef]

14. Yoon, J.; Li, C.C.; Shahar, D.; Tsui, D.C.; Shayegan, M. Wigner Crystallization and Metal-Insulator Transition of Two-Dimensional Holes in GaAs at $B=0$. Phys. Rev. Lett. 1999, 82, 1744-1747. [CrossRef]

15. Simmons, M.Y.; Hamilton, A.R.; Pepper, M.; Linfield, E.H.; Rose, P.D.; Ritchie, D.A.; Savchenko, A.K.; Griffiths, T.G. Metal-Insulator Transition at $B=0$ in a Dilute Two Dimensional GaAs-AlGaAs Hole Gas. Phys. Rev. Lett. 1998, 80, 1292-1295. [CrossRef]

16. Kane, B.E.; Pfeiffer, L.N.; West, K.W. High mobility GaAs heterostructure field effect transistor for nanofabrication in which dopant-induced disorder is eliminated. Appl. Phys. Lett. 1995, 67, 1262-1264. [CrossRef]

17. Huang, J.; Novikov, D.S.; Tsui, D.C.; Pfeifer, L.N.; West, K.W. Two-Dimensional Holes in GaAs HIGFETs: Fabrication Methods and Transport Measurements. Int. J. Mod. Phys. B 2007, 21, 1219-1227. [CrossRef]

18. Ando, T.; Fowler, A.B.; Stern, F. Electronic properties of two-dimensional systems. Rev. Mod. Phys. 1982, 54, 437-672. [CrossRef]

19. Noh, H.; Lilly, M.P.; Tsui, D.C.; Simmons, J.A.; Pfeiffer, L.N.; West, K.W. Linear temperature dependence of conductivity in the apparent insulating regime of dilute two-dimensional holes in GaAs. Phys. Rev. B 2003, 68, 241308. [CrossRef]

20. Huang, J.; Novikov, D.S.; Tsui, D.C.; Pfeiffer, L.N.; West, K.W. Nonactivated transport of strongly interacting two-dimensional holes in GaAs. Phys. Rev. B 2006, 74, 201302. [CrossRef]

21. Huang, J.; Xia, J.S.; Tsui, D.C.; Pfeiffer, L.N.; West, K.W. Disappearance of Metal-Like Behavior in GaAs Two-Dimensional Holes below $30 \mathrm{mK}$. Phys. Rev. Lett. 2007, 98, 226801. [CrossRef] [PubMed]

22. Spivak, B.; Kravchenko, S.V.; Kivelson, S.A.; Gao, X.P.A. Colloquium: Transport in strongly correlated two dimensional electron fluids. Rev. Mod. Phys. 2010, 82, 1743-1766. [CrossRef]

23. Pudalov, V.; Brunthaler, G.; Prinz, A.; Bauer, G. Breakdown of the anomalous two-dimensional metallic phase in a parallel magnetic field. Physica B 1998, 249, 697-700. [CrossRef]

24. Pillarisetty, R.; Noh, H.; Tutuc, E.; De Poortere, E.; Lai, K.; Tsui, D.; Shayegan, M. Coulomb drag near the metal-insulator transition in two dimensions. Phys. Rev. B 2005, 71, 115307. [CrossRef]

25. Kravchenko, S.; Sarachik, M. Metal-insulator transition in two-dimensional electron systems. Rep. Prog. Phys. 2003, 67, 1. [CrossRef]

26. Lu, T.M.; Li, Z.F.; Tsui, D.C.; Manfra, M.J.; Pfeiffer, L.N.; West, K.W. Cyclotron mass of two-dimensional holes in (100) oriented GaAs/AlGaAs heterostructures. Appl. Phys. Lett. 2008, 92. [CrossRef]

27. Zhu, H.; Lai, K.; Tsui, D.; Bayrakci, S.; Ong, N.; Manfra, M.; Pfeiffer, L.; West, K. Density and well width dependences of the effective mass of two-dimensional holes in (100) GaAs quantum wells measured using cyclotron resonance at microwave frequencies. Solid State Commun. 2007, 141, 510-513. [CrossRef]

28. Goldman, V.J.; Santos, M.; Shayegan, M.; Cunningham, J.E. Evidence for two-dimentional quantum Wigner crystal. Phys. Rev. Lett. 1990, 65, 2189-2192. [CrossRef]

29. Levy, L.; Dolan, G.; Dunsmuir, J.; Bouchiat, H. Magnetization of mesoscopic copper rings: Evidence for persistent currents. Phys. Rev. Lett. 1990, 64, 2074. [CrossRef]

30. Grüner, G. The dynamics of charge-density waves. Rev. Mod. Phys. 1988, 60, 1129-1181. [CrossRef]

31. Noh, H.; Lilly, M.P.; Tsui, D.C.; Simmons, J.A.; Hwang, E.H.; Das Sarma, S.; Pfeiffer, L.N.; West, K.W. Interaction corrections to two-dimensional hole transport in the large- $\mathrm{r}_{S}$ limit. Phys. Rev. B 2003, 68, 165308. [CrossRef]

32. Lai, K.; Pan, W.; Tsui, D.C.; Lyon, S.; Mühlberger, M.; Schäffler, F. Linear temperature dependence of the conductivity in Si two-dimensional electrons near the apparent metal-to-insulator transition. Phys. Rev. B 2007, 75, 033314. [CrossRef]

33. Jiang, H.W.; Stormer, H.L.; Tsui, D.C.; Pfeiffer, L.N.; West, K.W. Magnetotransport studies of the insulating phase around $v=1 / 5$ Landau-level filling. Phys. Rev. B 1991, 44, 8107-8114. [CrossRef]

34. Williams, F.I.B.; Wright, P.A.; Clark, R.G.; Andrei, E.Y.; Deville, G.; Glattli, D.C.; Probst, O.; Etienne, B.; Dorin, C.; Foxon, C.T.; et al. Conduction threshold and pinning frequency of magnetically induced Wigner solid. Phys. Rev. Lett. 1991, 66, 3285-3288. [CrossRef] [PubMed] 
35. Qiu, R.L.J.; Gao, X.P.A.; Pfeiffer, L.N.; West, K.W. Connecting the Reentrant Insulating Phase and the Zero-Field Metal-Insulator Transition in a 2D Hole System. Phys. Rev. Lett. 2012, 108, 106404. [CrossRef] [PubMed]

36. Andrei, E.Y.; Deville, G.; Glattli, D.C.; Williams, F.I.B.; Paris, E.; Etienne, B. Observation of a Magnetically Induced Wigner Solid. Phys. Rev. Lett. 1988, 60, 2765-2768. [CrossRef] [PubMed]

37. Chen, Y.P.; Sambandamurthy, G.; Wang, Z.H.; Lewis, R.M.; Engel, L.W.; Tsui, D.C.; Ye, P.D.; Pfeiffer, L.N.; West, K.W. Melting of a 2D quantum electron solid in high magnetic field. Nat. Phys. 2006, 2, 452-455. [CrossRef]

38. Zhu, H.; Chen, Y.P.; Jiang, P.; Engel, L.W.; Tsui, D.C.; Pfeiffer, L.N.; West, K.W. Observation of a Pinning Mode in a Wigner Solid with $v=1 / 3$ Fractional Quantum Hall Excitations. Phys. Rev. Lett. 2010, 105, 126803. [PubMed]

39. Paalanen, M.A.; Willett, R.L.; Littlewood, P.B.; Ruel, R.R.; West, K.W.; Pfeiffer, L.N.; Bishop, D.J. rf conductivity of a two-dimensional electron system at small Landau-level filling factors. Phys. Rev. B 1992, 45, 11342-11345. [CrossRef]

40. Jang, J.; Hunt, B.M.; Pfeiffer, L.N.; West, K.W.; Ashoori, R.C. Sharp tunnelling resonance from the vibrations of an electronic Wigner crystal. Nat. Phys. 2017, 13, 340. [CrossRef]

41. Kravchenko, S.V.; Perenboom, J.A.A.J.; Pudalov, V.M. Two-dimensional electron solid formation in Si inversion layers. Phys. Rev. B 1991, 44, 13513-13518. [CrossRef]

42. Pudalov, V.M.; D'Iorio, M.; Kravchenko, S.V.; Campbell, J.W. Zero-magnetic-field collective insulator phase in a dilute 2D electron system. Phys. Rev. Lett. 1993, 70, 1866-1869. [CrossRef] [PubMed]

43. Brussarski, P.; Li, S.; Kravchenko, S.; Shashkin, A.; Sarachik, M. Transport evidence for a sliding two-dimensional quantum electron solid. Nat. Commun. 2018, 9, 3803. [CrossRef] [PubMed]

44. Lee, P.A.; Rice, T.M. Electric field depinning of charge density waves. Phys. Rev. B 1979, 19, 3970-3980. [CrossRef]

45. Yoshioka, D.; Fukuyama, H. Charge Density Wave State of Two-Dimensional Electrons in Strong Magnetic Fields. J. Phys. Soc. Jpn. 1979, 47, 394-402. [CrossRef]

46. Anderson, P. Basic Notions of Condensed Matter Physics; Benjamin/Cummings: Menlo Park, CA, USA, 1984.

47. Aoki, H. Effect of coexistence of random potential and electron-electron interaction in two-dimensional systems: Wigner glass. J. Phys. C 1979, 12, 633. [CrossRef]

48. Chakravarty, S.; Kivelson, S.; Nayak, C.; Voelker, K. Wigner glass, spin liquids and the metal-insulator transition. Philos. Mag. Part B 1999, 79, 859-868. [CrossRef]

49. Chitra, R.; Giamarchi, T. Zero field Wigner glass. J. Phys. IV France 2005, 131, 163-166. [CrossRef]

50. Imada, M.; Takahashi, M. Quantum Monte Carlo Simulation of a Two-Dimensional Electron System-Melting of Wigner Crystal-. J. Phys. Soc. Jpn. 1984, 53, 3770-3781. [CrossRef]

51. Clark, B.K.; Casula, M.; Ceperley, D.M. Hexatic and Mesoscopic Phases in a 2D Quantum Coulomb System. Phys. Rev. Lett. 2009, 103, 055701. [CrossRef]

52. Knighton, T.; Wu, Z.; Huang, J.; Serafin, A.; Xia, J.; Pfeiffer, L.; West, K. Evidence of two-stage melting of Wigner solids. Phys. Rev. B 2018, 97, 085135. [CrossRef]

53. Millis, A.J.; Littlewood, P.B. Radio-frequency absorption as a probe of the transition between the Wigner crystal and the fractionally quantized Hall state. Phys. Rev. B 1994, 50, 17632-17635. [CrossRef]

54. Halperin, B.I.; Nelson, D.R. Theory of Two-Dimensional Melting. Phys. Rev. Lett. 1978, 41, 121-124. [CrossRef]

55. Nelson, D.R.; Halperin, B.I. Dislocation-mediated melting in two dimensions. Phys. Rev. B 1979, 19, $2457-2484$. [CrossRef]

56. Nelson, D.R.; Halperin, B.I. Solid and fluid phases in smectic layers with tilted molecules. Phys. Rev. B 1980, 21, 5312-5329. [CrossRef]

57. Young, A.P. Melting and the vector Coulomb gas in two dimensions. Phys. Rev. B 1979, 19, 1855-1866. [CrossRef]

58. Spivak, B.; Kivelson, S.A. Phases intermediate between a two-dimensional electron liquid and Wigner crystal. Phys. Rev. B 2004, 70, 155114. [CrossRef]

59. Kosterlitz, J.; Thouless, D. Long range order and metastability in two dimensional solids and superfluids. (Application of dislocation theory). J. Phys. C 1972, 5, L124. [CrossRef]

(C) 2018 by the authors. Licensee MDPI, Basel, Switzerland. This article is an open access article distributed under the terms and conditions of the Creative Commons Attribution (CC BY) license (http:/ / creativecommons.org/licenses/by/4.0/). 